\title{
Linx
}

Revue des linguistes de l'université Paris X Nanterre

$51 \mid 2004$

Théories de l'écriture et pratiques scolaires

\section{Constitution d'un grand corpus de textes d'élèves. Problèmes méthodologiques et premiers résultats}

\section{Marie-Laure Elalouf}

\section{(2) OpenEdition}

1 Journals

Édition électronique

URL : http://journals.openedition.org/linx/205

DOI : 10.4000/linx.205

ISSN : 2118-9692

Éditeur

Presses universitaires de Paris Nanterre

\section{Édition imprimée}

Date de publication : 1 décembre 2004

Pagination : $129-146$

ISSN : 0246-8743

\section{Référence électronique}

Marie-Laure Elalouf, «Constitution d'un grand corpus de textes d'élèves. Problèmes méthodologiques et premiers résultats », Linx [En ligne], 51 | 2004, mis en ligne le 28 janvier 2011, consulté le 10

décembre 2020. URL : http://journals.openedition.org/linx/205 ; DOI : https://doi.org/10.4000/linx.205

Département de Sciences du langage, Université Paris Ouest 


\title{
Constitution d'un grand corpus de textes d'élèves Problèmes méthodologiques et premiers résultats
}

\author{
Marie-Laure Elalouf \\ IUFM de Versailles - UMR 7114 CNRS-Paris X, MoDyCo
}

Une revue des travaux des vingt dernières années sur l'étude des textes d'enfants fait apparaittre l'absence de corpus conséquent réunissant pour plusieurs classes l'ensemble des textes produits par des élèves au cours d'une séquence d'enseignement, accompagnés des informations permettant d'en restituer le contexte ${ }^{1}$. Par ailleurs, la rareté des corpus longitudinaux suivant une cohorte d'élèves sur plusieurs années est mentionnée. Ce constat a conduit une équipe de l'IUFM de Versailles, réunissant des enseignants-chercheurs et des formateurs à constituer une base de données de textes d'élèves avec leur contexte d'enseignement, à l'appui d'une étude des relations complexes entre des scénarios didactiques mis en oeuvre par des enseignants particuliers et les possibles effets d'apprentissage que révèle l'évolution des textes écrits par leurs élèves.

La conception de ce corpus repose sur une hypothèse didactique articulée autour de quatre propositions : pour choisir un dispositif d'écriture pertinent dans une situation donnée, un enseignant doit pouvoir :

- mettre en relation une analyse du contexte d'enseignement, la définition d'un objectif d'enseignement-apprentissage et de tâches articulées entre elles dans les domaines de la lecture, de l'écriture, de l'oral et de l'étude de la langue ;

- anticiper sur les effets attendus, en tenant compte de l'hétérogénéité de la classe et de l'effet amplifiant, réducteur ou neutre de la démarche retenue ;

- choisir des modes d'intervention susceptibles de faire évoluer les textes d'élèves, d'un premier état à une version jugée définitive ;

\footnotetext{
${ }^{1}$ On se reportera à la récente revue de travaux de Jacques David dans FABRE-COLS, C., dir., 2000, Apprendre à lire des textes d'enfants, Boeck-Duculot, Bruxelles. Il existe toutefois des travaux se rapprochant de ce que nous cherchons à constituer concernant une classe (cf. BONNET, C. \& HUSER, N., 1996, Apprendre à écrire en $6^{e}$. Lausanne, Centre vaudois de recherches pédagogiques; BONNET, C. \& HUSER, N., 1998, Apprendre à écrire en $8_{e}^{e}$ Lausanne, CVRP.
} 
- inscrire son action dans une perspective à plus long terme, celle du développement des compétences d'écriture, à l'école et hors de l'école.

La constitution d'un tel corpus soulève des questions méthodologiques que nous tenterons d'exposer avant de présenter les choix opérés. Les pratiques d'enseignement ne produisent d'éventuels effets d'apprentissage qu'à travers toute une série de médiations. Entre le scénario de la séance et les écrits des élèves, fonctionnent comme autant de filtres le discours de l'enseignant, les interactions, les rapports singuliers à la langue et à l'écrit qui s'instaurent dans la classe. Ces médiations renforcent ou limitent les effets attendus, et ce de façon différenciée selon les élèves. Le recueil de données n'a donc pas prioritairement pour but d'établir des corrélations mais de reconstituer des dynamiques - celle du déroulement et de l'enchaînement des séances, celle des différents états des textes - et de cerner les différents niveaux d'interaction. C'est la raison pour laquelle, dans un premier temps, l'analyse interne de chaque séquence a été privilégiée ${ }^{2}$.

En constituant un recueil de séquences d'écriture réalisées à divers niveaux dans des conditions différentes, il s'agissait de mieux cerner la spécificité de chacune non seulement à travers son analyse interne, mais aussi par une étude contrastive sans être normative: entreprise plus délicate dont j'essaierai de donner un aperçu en choisissant l'exemple de la description, composante textuelle explicitement ou implicitement requise selon les consignes dans plusieurs séquences du CM2 à la $5^{\mathrm{e}}$ et prenant des formes différentes selon le rapport à l'écrit instauré par l'enseignant et le dispositif didactique adopté ${ }^{3}$.

\section{La base de données : méthodologie}

\section{Quelles données retenir?}

Composée de huit séries d'observations, la base n'a pas les caractéristiques d'un échantillon représentatif mais d'un recueil méthodique, selon des règles définies à l'avance. Pour ne porter aucun jugement de valeur a priori sur les pratiques observées, nous avons retenu tout enseignant acceptant d'accueillir dans sa classe un chercheur pour assister à la totalité d'une séquence et recueillir les écrits des élèves. Nous écartions certes, de fait, ceux qui refusaient l'accès de leur classe ou la communication des travaux de leurs élèves.

Le choix du niveau d'enseignement - la charnière CM2-collège - repose sur une hypothèse communément partagée : entre l'école et le collège, les conditions, les modalités et les enjeux des activités d'écriture changent. La confrontation de données recueillies dans la dernière classe de l'école primaire et au début du collège devrait permettre de cerner plus précisément des ruptures, des continuités et des sources possibles de malentendus chez les élèves de collège, en se gardant de généralisations hâtives compte tenu de la taille du corpus.

\footnotetext{
${ }^{2}$ Cf. articles de C. Boré, M.-L. Elalouf \& J. Keraven en bibliographie.

${ }^{3}$ Une présentation des résultats de recherche a été rédigée à l'attention des formateurs d'enseignants : ELALOUF M.-L., dir., à paraître en 2004, Écrire entre 10 et 14 ans, un corpus, des analyses, des repères pour la formation. CRDP de Versailles.
} 
La difficulté à trouver une unité d'observation commune à l'école et au collège est à cet égard emblématique: au collège, l'unité administrative est le cours de 55 minutes et l'unité didactique la séquence telle que la définissent les programmes de 1996: "mode d'organisation des activités qui rassemble des contenus d'ordre différent autour d'un même objectif, sur un ensemble de plusieurs séances » (Français, Programmes p. 31). Les programmes de l'école primaire appellent à la mise en oeuvre de " projets d'écriture » en relation avec la lecture et d'autres activités ; ils insistent aussi sur l'écriture au quotidien comme mode d'élaboration progressive des connaissances. Nous avons souhaité n'exclure aucune de ces dimensions en retenant pour unité ce que nous avons appelé « séquence », avec une définition susceptible de rendre compte de pratiques observées dans le premier et le second degré : ensemble de moments d'enseignement-apprentissage, appelés "séances", organisés autour d'un objectif d'apprentissage et mettant en jeu différentes pratiques langagières, oral, lecture, écriture.

Dans l'impossibilité de déterminer à l'avance des éléments du contexte qui seraient susceptibles de juger un rôle, il a semblé préférable de relever tout ce qui pouvait aider à comprendre, en les situant, les observations recueillies :

- informations, toutes anonymées, sur l'établissement, la classe et l'enseignant, notamment son rapport à l'écrit.

- description précise de chaque séance avec consignes, démarches, supports ;

- enregistrement soutenu par une prise de notes et quand cela a été possible enregistrements vidéo.

Les écrits des élèves forment le cœur de la base de données. Le choix a été fait de l'exhaustivité en fournissant tous les textes produits dans la séquence, dans leurs différents états, avec les interventions du professeur sur ces textes. Quand la situation le permettait, ont été recueillies des productions antérieures ou à échéance plus lointaine, pour analyser les évolutions à moyen terme. Ainsi la séquence A comporte, outre les textes produits par toute la classe dans une séquence de début d'année, un suivi longitudinal de 6 élèves avec les évaluations CE2 et $6^{\mathrm{e}}$ ainsi que les 32 textes écrits au cours de l'année de CM2 dans leurs différentes versions.

La mise en relation de ces différents éléments montre qu'au-delà du synopsis de la séquence, c'est une analyse fine de la pratique professionnelle qui donne accès à des hypothèses sur les effets d'apprentissage. L'enseignante qui a proposé la séquence A, par exemple, a une formation universitaire en lettres et en langues, elle s'intéresse aux pratiques culturelles de la lecture, a suivi des ateliers d'écriture, appartient à une société d'amis d'écrivain. Et c'est tout naturellement qu'elle propose aux élèves de descendre dans la cour après la récréation pour y prendre des notes avant d'écrire un paragraphe descriptif. Elle a sans doute en mémoire les carnets que certains écrivains portent sur eux et elle se met à écrire, ses élèves sachant qu'ils pourront ensuite lire ses textes. Pour ceux-ci, la situation est bien moins « naturelle » : qu'y a-t-il d'intéressant à noter dans une cour de récréation sans animation ? Que deviendront ces notes ? À qui sont-elles destinées? Et pourtant, comme entraînés dans une expérience où la personnalité de l'enseignante joue un rôle majeur, tous écrivent une première version, alors qu'on pouvait escompter des refus. Ils passent de simples listes à une mise en phrases, tel Henri : 
Marie-Laure Elalouf

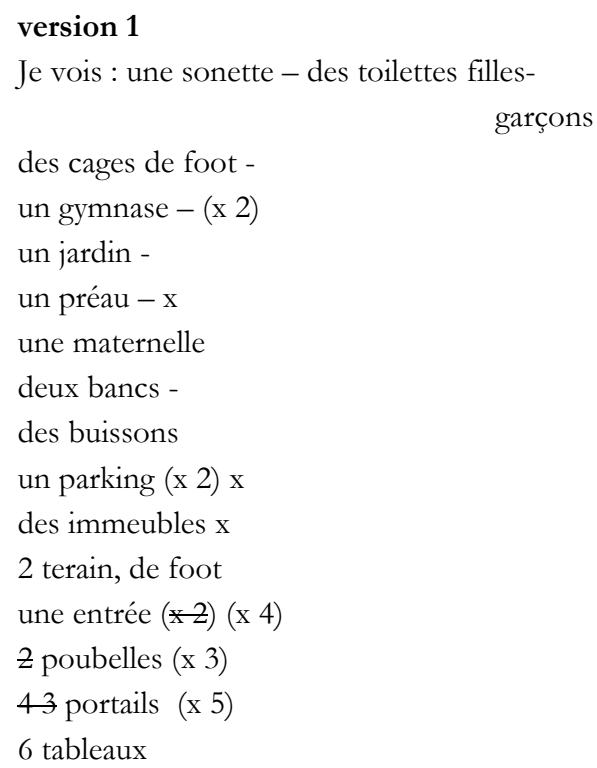

\section{version 2}

$<$ je vois $>$

Dans la <cour $>$ y a: une sonnette, des toilettes (filles et garçons), 2 cages de foot, 2 gymnase, un jardin, un préau, une maternelle, des bancs, des buisons deux parking, des immeubles, 2 deux terrains de foot (1 petit, 1 grand), 4 entrée, 3 poubelles, 5 portails, des tableaux, des arbres et des feuilles jaune (mortes).

Le résultat ne correspond cependant pas aux attentes de l'enseignante, qui pour certaines sont énoncées - se conformer aux normes stylistiques de l'écriture scolaire et pour d'autres restent implicites - suivre le modèle de la description littéraire. Il en résulte un échange oral :

M1 : certains font des énumérations et des répétitions $>$ comment pourrait-on éviter ça $<$

[M écrit au tableau un exemple pris chez. un élève: "Je vois un parking, des voitures, un blouson, deux cages de foot ». ]

M2 : qui pourrait me proposer de transformer cette phrase en plusieurs phrases $>$ On pourrait garder le début: je vois un parking. $>$ Que pourrait-on écrire sur les voitures $<$

E1: je vois des voitures $>$

M3: Oui mais tu répètes je vois $>$

E2 : il y a des voitures $>$

M4 : oui, mais on a doit de ne pas employer il y $a>$ commence ta phrase par des voitures //

E3: //

M5 : que font les voitures < 
E4 : des voitures sont garées sur le parking >

M6 : oui / et comment faire pour ne pas répéter parking <

E5 : des voitures sont garées dessus.

M7 : [oui intonation d'insistance. Elle écrit : Dessus, sont garées des voitures.]

Guidés pas à pas, les élèves admettent les interdits, proposent un «verbe d'action» (au passif) mais n'abordent pas le sens de ces transformations, ni l'organisation textuelle qui les rendrait possibles. Pourtant certaines versions finales ne sont pas de simples juxtapositions de phrases. Henri simule le chemin du regard grâce à des indicateurs de lieu en position thématique ou rhématique; Pauline propose une évocation fondée d'abord sur la métonymie puis sur des associations sensorielles. Toutes choses que l'enseignante n'avait sans doute pas prévues mais qui influeront sur ses choix ultérieurs - par exemple, la prise de notes sur la fête foraine dans la perspective du compte rendu - ou la rendront sensible à des compétences non travaillées en classe mais apparues dans les écrits d'élèves, et exploitées ensuite dans les travaux de création poétique.

\section{2. Comment transcrire les données?}

\section{2. 1. Les corpus oranx}

Les nombreux travaux sur des corpus oraux nous invitaient à rejeter une transcription-réécriture selon le code de l'écrit, le confort de lecture ne se gagnant qu'au prix de choix discutables.

Nous tournant vers les codes déjà utilisés en linguistique de corpus, il nous est apparu qu'il fallait tenir compte de deux contraintes :

- assurer la lisibilité tout en respectant la spécificité de la langue parlée (Benveniste, C.-B., 1997)

- rendre compte des phénomènes de lecture/dictée dans le cadre de l'écriture coopérative.

Par exemple, l'échange suivant peut s'interpréter de deux façons selon que l'élève répond à la question ou comprend qu'on attend de lui une autre tournure de phrase :

M6 : que font les voitures <

E4: des voitures sont garées sur le parking $>$

ou

M6 : que font les voitures <

[E4 dictant $]$ : des voitures sont garées sur le parking $>$.

Empruntées respectivement, pour l'esprit sinon la lettre, à F. Gadet (1989) et M.-M. de Gaulmyn (1998), ces conventions restent relativement grossières, compte tenu de l'objectif poursuivi, centré sur les textes d'élèves. Les commentaires sont apportés selon les mêmes conventions que celles adoptées pour l'écrit de façon à ne pas multiplier les codes: le passage commenté est encadré de crochets et le 
commentaire suit le mot commenté mais s'en distingue par son corps, plus petit, et par l'italique.

\section{2. 2. Les corpus écrits}

Le support électronique permet de restituer le texte d'élève avec ses ratures, ses ajouts ainsi que les commentaires, annotations et interventions du professeur sur celui-ci, selon des couleurs et des codes différents. Mais l'analyse exige que la copie scannée, document brut, soit accompagnée de transcriptions.

Quand les modifications apportées au texte sont le fait de son auteur, ce qui est le cas des brouillons, la version iconique est la plus proche du texte produit: en effet, elle restitue scrupuleusement la graphie, la mise en page, les ratures (passages barrés), les ajouts (passages entre soufflets), à la suite ou au-dessus selon que l'ajout est contemporain du moment de l'écriture ou postérieur à celui-ci. C'est ce que l'on observe en 1. 1.

Mais parfois, les qualités du texte sont occultées par des choix graphiques. On peut alors ajouter, à la suite de Clairelise Bonnnet (1994), une version conforme à la norme graphique mais respectant les choix de ponctuation et de typographie des élèves, comme ici :

$6^{\mathrm{e}}$ : écrire une fin heureuse ou malheureuse au conte Le joueur de flùte de Hamelin

Alors le joueur de flûte quitta la ville et tous les enfants le suivirent.

Ils partirent par les routes et finirent par arriver dans une abbaye pour les chants. Donc à la fin de l'année, les habitants de Hamelin sont venus voir les chœurs et un des parents reconnut sa fille.[illisible] tous les habitants reconnurent leurs enfants et le chef d'orchestre était le joueur de flûte bien déguisé et ensuite il avait installé une grande trappe et il fila à l'autre bout du monde. Ils atterrirent en Chine. (Amaury, $6^{\mathrm{e}}$ )

Pour rendre compte à la fois des choix graphiques de l'élève et des annotations du professeur, la version iconique ne suffit pas: il est nécessaire de décrire les incursions d'une voix autre dans le texte initial et de délimiter les portions de texte concernées, généralement limitées à un mot ou un syntagme comme l'avait déjà noté C. Masseron (1989).

Alors le joueur de flûte quitta la ville et tous les enfants le suivirent.

Ils partirent par les routes et finirent par arriver dans une abbaye pour les [chans souligné par $P$, ? dans la marge]. [...][le biffé par P, -1 surchargé par P en $-\mathrm{L}]$ chef d'orchestre était le [-s biffé par $P$, au-dessous inscription de $P$ n'était autre que] le joueur de flûte bien déguisé, [et ensuite biffé par $P$, comme en marge] il avait installé une grande trappe [et biffé par $P$ ], il fila à l'autre bout du monde. [Il flèche de $P$, en dessous annotation le musicien seul ?] [attairirent souligné deux fois par $P$, flèche de $P$ annotation de $P$ en dessous qui ?] en Chine. [annotation de P et les enfants?]

Note 8/20 Appréciation: fin maladroite.

D'une lecture moins fluide, cette version partielle à deux voix rend compte de la perception qu'a un professeur d'un texte d'élève quand il le découvre en le 
corrigeant pas à pas. De «maladroite » pour le professeur, la fin devient pathétique lorsqu'on la découvre sans ses scories. L'intensité dramatique croît d'étape en étape : l'enchantement des enfants qui non seulement suivent le musicien mais unissent leurs voix en des chœurs qu'il dirige, la cruauté de l'invitation des parents au spectacle, le premier coup de théâtre (un des parents reconnut sa fille), suivi d'un second laissant espérer une libération (tous les habitants reconnurent leurs enfants) et l'ultime stratagème du joueur de flûte, qui devant les parents impuissants, met en scène la disparition définitive de leurs enfants.

\subsection{Comment organiser les données?}

La conception de l'arborescence a tenu compte de trois exigences :

- donner à tout moment accès aux principes de constitution de la base et aux références théoriques qui la fondent;

- présenter chaque séquence dans son unité ;

- permettre, grâce à des liens hypertextuels, des mises en relations au sein d'une séquence et le cas échéant d'une séquence à l'autre.

Pour chacune des huit séquences, les données sont présentées de la façon suivante :
10. Contexte
20. Séquence
30. Textes
40. Analyses
50. Annexes

\section{Perspectives d'exploitation de la base de données}

Une lecture transversale des données fait apparaitre différentes réponses à des problèmes d'apprentissage, qu'il s'agisse de la morphologie, du lexique, de la syntaxe, de l'énonciation, du texte vu sous le double angle de sa cohérence interne et de son inscription dans une culture. Selon le professeur, la classe, le moment de l'année, les priorités diffèrent, la part dévolue à l'implicite et à l'explicite se déplace. Toutefois, les comparaisons sont délicates et le chemin encore long avant une possible modélisation. Aussi préférerons-nous ici l'étude détaillée d'une pratique courante, se démarquant parfois à peine de la traditionnelle rédaction et faisant appel à des modèles issus de la culture littéraire des professeurs: l'insertion de la description dans le récit. Cet exercice d'écriture figure de façon explicite dans les programmes de $6^{\mathrm{e}}$ et implicite dans les programmes de cycle 3 et de cycle central $\left(5^{\mathrm{e}}-4^{\mathrm{e}}\right)^{4}$.

\footnotetext{
${ }^{4}$ Cycle 3 (CE2-CM2) : « élaborer et écrire un récit d'au moins une vingtaine de lignes » Programmes 2002 pour l'école élémentaire, p. 194 ;

$6^{e}:$ : insertion dans une narration de courtes notations descriptives », programmes de 1996, p. 20 ; $5^{\mathrm{e}}:$ « récit complet rendant compte d'une expérience personnelle, récit fictif ayant un rapport avec le Moyen âge »;
} 
Plusieurs études ont montré que la pratique de la description n'allait pas de soi pour les élèves jusqu'à l'entrée au collège : souvent, le référent est supposé connu du lecteur, tout au plus est-il rapidement caractérisé lors de sa première introduction. Mais l'idée que celui-ci soit susceptible d'évoluer grâce aux dénominations ou que la description fasse progresser le récit ne s'impose que lentement. Lorsque la description est attendue par le professeur, par référence à des modèles littéraires, mais non explicitement demandée dans la consigne ni travaillée au préalable, on observe des résultats, proches de ceux obtenus dans la classe de 6e précédemment évoquée.

Niveau : 6e, début d'année (séquence G)

Lieu : 78

Objectif : Ecrire une suite de récit

Consigne : écrire la fin heureuse ou malheureuse du conte Le joueur de flùte de Hamelin.

\section{Activité attendue :}

- s'appuyer sur l'interprétation du conte pour concevoir une fin plausible ;

- être attentif à la désignation des référents : cohésion, caractérisation ;

- respecter des normes de présentation, de lisibilité et de stylistique scolaires

Résultats (sur 29 élèves, abréviation : $\mathrm{E}$ )

- insertion d'une séquence descriptive : $3 \mathbf{E}$

C'était magique, tous les enfants dansaient, chantaient, criaient tout en avançant! (Maud, 16/20)

Une semaine plus tard, le joueur de flûte toujours avec les enfants passa dans des champs, dans une forêt, sur un grand pont. Ils aperçurent un village avec des enfants qui jouaient à la corde. (Romain, 06/20), (cf. Camille 07/20)

- insertion d'éléments descriptifs ponctuant l'évolution d'un référent (dominante du conte lu) : $11 \mathrm{E}$ (dont 4 réponses doubles)

- dénominations et caractérisations accompagnant l'évolution du référent :

Le joueur de flûte changea de musique, celle-ci fut lente et triste. Les enfants s'élevèrent vers le ciel (...) Les habitants d'Hamelin virent une ombre et remarquèrent qu'elle avait la forme d'une centaine d'enfants regroupés. (Gaétan 13 / 20)

- variation de dénomination sans réelle pertinence textuelle : le joueur de flutte ... le troubadour... le charmeur de rats laissa partir les enfants. (Solène 12 / 20)

- insertion d'un ou plusieurs éléments descriptifs accompagnant la première mention d'un référent : $14 \mathrm{E}$ (dont 4 réponses doubles)

la plus grande colline (Raphaël 12/20), une petite cabane abandonnée (Astrid, 08/20)

4e : «récit fictif ayant pour cadre le XVIIe, le XVIIIe ou le XIXe siècles ", Programme de 1996, pp. $72-73$. 
- absence d'éléments descriptifs : $\mathbf{5} \mathbf{E}$ (entre 07 et 09, une copie non notée).

Une minorité d'élèves éprouve le besoin d'insérer une séquence descriptive dans son récit, prenant le risque de rompre la progression de leur texte, risque parfois chèrement payé comme en témoignent certaines notes. Une majorité d'élèves insère des éléments descriptifs mais leur fonction est très variable : tantôt dictée par le récit lui-même (cf. Gaétan), tantôt purement ornementale (cf. Solène), rarement liée à un choix de dénomination significatif. La pratique la mieux attestée est l'insertion d'une rapide caractérisation lors de la première désignation du référent, caractérisation généralement stéréotypée. Sans qu'il soit possible d'établir une relation de causalité, on constate que les textes dénués d'éléments descriptifs sont les plus déficients aux yeux du professeur.

Face à ce constat, on trouve dans le corpus trois dispositifs alternatifs, à trois niveaux différents : une approche grammaticale ayant pour cadre la phrase en CM2, une approche fondée sur l'imitation d'un texte lu en $6^{\mathrm{e}}$ et une approche s'appuyant sur la délinéarisation de l'écriture narrative et l'explicitation des fonctions de la description en 5 e.

Niveau : CM2, fin d'année scolaire (séquence B)

\section{Lieu : Essonne}

Objectif : réinvestissement de connaissances grammaticales au cours de l'écriture d'un récit

Consigne : alors est-ce que maintenant vous pourriez penser à enrichir cela / si vous / vous avez dans votre phrase / il doit s'en trouver au moins un / un nom / est-ce que je peux pas donner un peu plus de détails / qu'est-ce que je peux écrire pour compléter un nom <

\section{Activité attendue :}

- relire la première phrase ;

- identifier un nom dont il serait pertinent de caractériser le référent ;

- ajouter une expansion du nom qui convienne au niveau syntaxique, sémantique et textuel.

\section{Résultats (sur $21 \mathrm{E}$ )}

- ajout d'une caractérisation adéquate : $6 \mathrm{E}$

Harry [qui fait des galipettes sur son balaie ajouté avec un becquet] est impatient que ça soit le repas d'Halloween.

- inadéquation syntaxique : $4 \mathbf{E}$

Draco Malfoy décut par la défaite de serpentard fut au bureau du directeur [pendant son travail manuel ajouté dans un becquet ]

Harry Potter [Repas de Halloween que ce grand parent lui a apporté barré par l'élève, croix à l'initiale par le professeur, remplacé au-dessus par Le repas de Halloween que Hagrid lui apporte.] 
Marie-Laure Elalouf

- inadéquation sémantique et/ou textuelle : $1 \mathbf{E}$

Harry Potter [qui a des-est blond : ajouté dans un becquet] veut aller à la kermesse (...)

- absence d'ajout ou suppression de caractérisation : $10 \mathrm{E}$

Harry est heureux d'avoir gagné le match de Quidditch [mais barré] Rom lui apprend que Serpentard veut une revanche.

Harry Potter [portant cette foi journés Une tenu de sorcier bleu marine ajouté dans un becquet puis barré par l'élève] ayant bien joué la dernière fois.

Les élèves viennent d'écrire la première phrase de leur texte. Ils sont invités à la relire pour apporter une caractérisation. Mais il ne s'agit pas pour eux d'apporter une information indispensable à la poursuite du récit en se projetant dans la fiction qu'ils élaborent. Les vocables choisis dans la consigne relèvent des classes de mots (un nom), de la syntaxe (compléter un nom) et de la sémantique sous une formule impressionniste couramment utilisée (enrichir un nom) mais porteuse d'une double confusion : entre le nom et le référent, entre la syntaxe et la sémantique (ce que permet la polysémie du verbe compléter). Cette confusion est entretenue par le choix du mot détails, lui aussi couramment employé dans les leçons sur l'expansion du nom, mais également à propos des compléments circonstanciels, ce qui induit un autre malentendu (ajout de pendant son travail manue). La polysémie du mot détail elle-même vient interférer: soit on considère un ensemble à travers ses éléments et il est nécessaire d'entrer dans les détails, soit on ne retient que le caractère non essentiel des détails et ils deviennent négligeables.

Un paradoxe apparaît entre l'étroit guidage proposé par l'enseignante et les résultats peu probants. Tout investis dans un discours qu'ils commencent à élaborer, les élèves sont appelés à prendre pour objet la phrase qu'ils viennent d'écrire: décentrement que peut opérer fructueusement un scripteur expert mais qui ne semble possible que pour environ un élève sur quatre dans cette classe de CM2. À moins peut-être de dissocier explicitement les deux phases et de traiter, en observation réfléchie de la langue, la détermination au sens large, à partir de textes d'élèves où certains référents, évidents pour le scripteur, sont difficilement identifiables pour les lecteurs, élèves ou professeur.

À l'opposé de la volonté d'explicitation illustrée ci-dessus, l'imitation, vecteur d'une grammaire implicite, a longtemps été pratiquée. Faire observer, imiter une séquence descriptive est ce qui est proposé aux élèves qui écrivent ensuite un texte sur un événement qui les a marqués (la tempête de décembre 1999), en suivant la structure syntaxique d'un texte de Gide présenté sous cette forme, après restitution des formes verbales, toutes à l'infinitif :

Quand la rivière commença à monter

Il y en eut qui se réfugièrent sur la montagne;

D'autres qui se dirent : le limon engraissera nos champs ;

D'autres qui se dirent : c'est la ruine ;

D'autres qui ne se dirent rien du tout.

Quand la rivière eut bien monté, 
Il y avait des endroits où l'on voyait des arbres,

D'autres où l'on voyait des toits de maisons,

Des clochers, des murs et plus loin des collines; d'autres endroits où l'on ne voyait rien du tout.

Il y avait des paysans qui faisaient monter leurs troupeaux sur les collines ;

D'autres qui emportaient dans un bateau leurs petits enfants.

\section{André Gide}

Niveau : 6e milieu d'année (séquence $G$ )

Lieu : 78

Objectif : s'appuyer sur la structure d'un texte littéraire pour mettre en forme une expérience réelle ou fictive

Consigne : tout l'exercice de rédaction va être de s'intéresser surtout à l'ordre des phrases et puis au vocabulaire // on va passer du texte de Gide qui est surtout une inondation [au] vocabulaire de la tempête $>$

[observation syntaxique et stylistique du texte de Gide]

vous vous représentez dans votre tête les scènes de tempête que vous avez vues ou lues et vous les écrivez au fur et à mesure >

\section{Activité attendue :}

- écrire un texte descriptif avec une progression à thèmes dérivés et une opposition premier plan / second plan;

- respecter des contraintes syntaxiques tout en concevant une autre cohérence sémantique ;

- jouer sur les contrastes entre différentes manifestations d'un même événement, source de différents points de vue et comportements ;

- écrire deux jours après un second texte sur un autre cataclysme pour un contrôle des acquis.

Résultats (sur 23 E, la plupart ayant écrit 2 textes)

- respect de la structure formelle avec recherche d'un effet à travers l'organisation et la chute de la description, l'opposition premier plan / second plan et les oppositions sémantiques : $\mathbf{1 4} \mathbf{E}$ (dont 8 pour leurs deux textes)

- non respect de la structure formelle mais recherche d'un effet par les mêmes moyens que précédemment : $\mathbf{1 0} \mathbf{E}$ (dont 4 pour leurs deux textes)

recherche d'un effet sur le texte à travers l'opposition premier plan / second plan et les oppositions sémantiques : $4 \mathbf{E}$ (pour un de leur texte seulement)

- dépassement de la structure formelle vers le conte fantastique, eschatologique ou la fable : $\mathbf{3} \mathbf{E}$ (dont une pour ses deux textes, Marie).

Les textes conformes aux attentes du professeur sont plus nombreux que dans le cas précédent, mais derrière cette réussite globale, des cas très différents se présentent: une majorité d'élèves confirment dans leur second texte les 
caractéristiques du premier. Les autres négocient l'adaptation de la structure au changement de thème imposé. Mais la référence au texte littéraire masque les tentatives d'adaptation des élèves. Dans son premier texte, Paul, par exemple, évoque la tornade comme un récit d'aventure : les pensées rapportées sont écartées au profit de verbes d'action et les constats finaux à l'imparfait au profit d'une chute au passé simple.

La tornade de $\mathrm{Nice}^{5}$

Quand le vent commença à souffler dans le port de Nice,

Il y avait des poissons et des dauphins qui s'envolaient.

Des gens s'enfuyaient,

D'autres qui étaient pris par la tornade,

D'autres qui étaient projetés sur les plages,

D'autres qui ne bougeaient plus.

Quand le vent ne souffla plus bien, on put voir

Apparaître un requin géant.

Il y avait des personnes qui s'enfuirent sur les plages,

D'autres dont la vie était en danger.

Des bateaux, des voitures et des motos étaient

coincées entre les dents du requin.

D'autres véhicules comme les avions

Bombardaient le requin qui ne sentit rien.

Il y avait des militaires qui ouvraient le feu,

Des tanks.

D'autres militaires partirent tout de suite. (Paul)

Note 5,5 / 10 Appréciation : quelle imagination. Mais était-ce bien le sujet?

Le second texte est beaucoup plus proche de la structure attendue, l'opposition premier plan / arrière plan mieux maîtrisée, mais la pause descriptive se déploie en petits tableaux, au détriment d'une conclusion sur la diversité des comportements humains face à la catastrophe, thème évoqué seulement dans la première partie, au discours indirect. Or ce refus de conclure est précisément un indice de la spécificité de la description par rapport au texte précédent, mal dégagé du schéma narratif. Ce que la note et les appréciations ne font guère apparaître, c'est la différence qualitative entre les deux textes.

\section{L'inondation du Sahara}

Quand le ciel commença à devenir noir dans le

\footnotetext{
5 Tous les textes de cette séquence sont présentés en orthographe normée, les élèves ayant généralement corrigé les erreurs signalées par le professeur.
} 
Sahara, une énorme pluie tomba.

Il y avait des villages où l'on croyait que

C'était la fin du monde.

D'autres qui étaient entièrement inondés et où

Les habitants se réfugiaient sur les toits.

D'autres qui étaient en fête et qui disaient que

C'était un don du ciel.

D'autres qui ne voyaient rien.

Quand la pluie cessa, il y avait des poissons partout.

Des varans, des vipères et autres serpents

Prenaient un bain.

Il y avait des poissons qui sautaient partout.

D'autres qui gobaient les sauterelles mortes

Qui flottaient à la surface.

\section{Note 6,5}

Il est à noter que les élèves les plus valorisés sont ceux qui dépassent la structure formelle en la pliant aux codes d'un genre différent de celui dont le texte de Gide est issu, telle Marie qui le fait d'emblée :

La lune de tempête

Quand la tempête commença à souffler,

Il y en eut qui rentrèrent chez eux à toute vitesse,

D'autres qui se cachèrent,

D'autres qui s'abritèrent sous leurs parapluies,

Mais personne ne regarda la lune.

Quand la tempête fut bien calmée, une deuxième lune apparut.

Il y en eut qui se dirent : "c'est la fin du monde!»

D'autres qui se dirent : «c'est vraiment bizarre ...»

D'autres qui ne se dirent rien du tout.

Il y eut une autre tempête moins forte la nuit suivante,

Et la deuxième lune disparut,

Et personne ne se posa de questions. (Marie)

Note $: 9 / 10$

D'une réflexion sur la diversité des comportements humains dans les situations extrêmes, elle tire une évocation de l'incapacité des hommes à lire des signes, par-delà les actes et les paroles convenus, et referme son texte sur une énigme. Il y a eu appropriation du texte, incorporation à une culture personnelle pour produire un écrit unique, littéraire, non une réplique de l'extrait de Gide. 
Mais ce qu'une élève comme Marie fait d'elle-même, sans consigne ni guidage particuliers, comment le susciter chez un plus grand nombre ? C'est à cette question que le troisième dispositif, expérimenté en $5^{\mathrm{e}}$ tente de répondre.

Niveau : 5 e (fin d'année)

Lieu : 91

Objectif : écrire un roman historique au terme de séquences ayant fait alterner initiation à la langue, la littérature et la culture du Moyen Age, recherches documentaires, lectures d'extraits de romans et de films historiques, réflexions progressives sur les fonctions de la description et son insertion dans le récit.

Consigne intermédiaire : vous allez travailler sur le principe du puzzle /parfois il faut deux trois morceaux pour faire une tête / / il s'agit de voir ce qu'on va décrire / quitte à ce qu'après dans le récit / on puisse éclater la description ou le récit comme dans le Chevalier au bouclier vert $>$ // vous allez faire des phrases ou des morceaux de phrases que vous pourrez reprendre dans l'ordre que vous voudrez $>$

Activité attendue :

- faire des descriptions partielles selon la fonction, l'importance des personnages et des lieux ;

- choisir le ou les lieux d'insertion de ces descriptions dans le roman historique; - faire évoluer ces descriptions en fonction du cours du récit et réciproquement, tirer parti de ces descriptions dans le récit.

Résultats (sur 4 groupes de 3 élèves représentatifs de la classe)

- insertion des fragments descriptifs sans modification, lors de la première mention du référent: ce procédé de «collage» caractérise les groupes 2 et 3 . L'insertion est parfois fluide, souvent problématique: disjonction sujet/verbe et incompatibilités sémantiques (G3), énumérations hétéroclites, ruptures dans la progression thématique $(\mathrm{G} 2)$.

\section{insertion des fragments descriptifs avec modification :}

réduction des fragments descriptifs au moment de leur insertion : Le groupe 1 part d'un énumération des parties du château qu'il transforme en phrase descriptive interrompant le récit (état 3) avant de ne retenir que quelques éléments s'intégrant dans le récit.

- éclatement des fragments descriptifs: le groupe 4 maintient les éléments descriptifs présents dans les premières versions mais travaille leur insertion.

- recomposition des fragments descriptifs :

apporter une clôture à la description : G1

mettre en relation personnage et lieu : G2

état 1 
Une jeune fille : une paysanne aux cheveux blonds et longs les yeux bleus avec une robe déchirée texte définitif une ferme abandonnée dans laquelle une jeune fille au cheveux blonds et longs, les yeux bleus, une robe déchirée, vivait

- modification radicale des fragments descriptifs :

changer de registre: le groupe 4 passe du burlesque au pathétique, du texte documentaire à un récit dramatique.

- suppression de fragments descriptifs :

G1 : la quasi totalité des fragments initiaux sont abandonnés pour la cohérence du récit ou en raison de difficultés à insérer des fragments expansés

G 2 et 3 : aucune

G 4 : une pour la cohérence du récit

\section{- ajouts de fragments descriptifs :}

G1 : de nombreux ajouts pour la cohérence du récit et la «couleur locale »

$\mathrm{G} 2$ : quelques notations psychologiques

G3 : des descriptions systématiques des personnages et des lieux

G4 : des ajouts très nombreux et variés pour la mise en intrigue.

Après avoir étudié les fonctions de la description dans un roman historique de littérature de jeunesse et des extraits de romans médiévaux, les élèves sont amenés à anticiper sur ce qu'ils donneront à voir. Le professeur pose des "passages obligés ». Or, l'observation des romans achevés fait apparaittre une variété beaucoup plus grande que celle prescrite : pris dans la dynamique de l'écriture longue, les élèves recomposent, suppriment peu mais ajoutent beaucoup, dans des proportions certes différentes. Des stratégies se dessinent:

- le groupe 1 ne retient que des fragments descriptifs brefs, en cohérence avec le récit et ajoute les détails nécessaires à la couleur locale, sans pause qui ralentirait le tempo du récit ;

- le groupe 2 plaque les descriptions sans toujours tenir compte de leur insertion et de leur compatibilité sémantique, mais recompose un passage après avoir découvert que l'on «avait le droit» de mettre en relation description des lieux et des personnages; quelques notations psychologiques sont ajoutées ;

- le groupe 3 multiplie les descriptions calibrées à l'identique, avec quelques difficultés d'insertion et quelques incohérences sémantiques, proposant une galerie de portraits, certes stéréotypée mais vaste ;

- le groupe 4 procède aux modifications et aux ajouts les plus importants, au service d'une mise en intrigue savamment menée, autour d'un paradoxe: rendre présente celle que l'on ne peut décrire puisqu'elle cache son identité sous le travestissement d'un ménestrel et jalonner le texte de suffisamment d'indices pour que la révélation finale soit crédible (Elalouf M-L \& Keraven, J., 2002 pour une analyse des textes). 
La confrontation de ces corpus ne cherche pas à établir quel dispositif est $a$ priori le plus pertinent, mais tente d'expliquer les écarts entre activité attendue et effets obtenus. Ainsi, dans la séquence $\mathrm{B}$ comme dans la séquence $\mathrm{H}$, les élèves sont guidés dans le choix des opérations de réécriture. Pourtant, seul un élève sur quatre introduit une expansion du nom pertinente, tandis qu'en $\mathrm{H}$, tous les élèves ajoutent de nouveaux fragments descriptifs, en cohérence avec la trame initiale, même si certains éprouvent des difficultés à insérer ceux qu'ils avaient déjà écrits. L'analyse fait apparaitre quatre causes majeures :

- l'exercice proposé en $\mathrm{B}$ ne se rattache pas à un projet d'écriture, tandis qu'en $\mathrm{H}$, les élèves ont déjà écrit le synopsis de leur roman, conçu la couverture et l'incipit ;

- en B, la consigne est donnée en cours d'écriture, sans autre explication qu'ornementale - il s'agit d'enricbir un nom - tandis qu'en $\mathrm{H}$, le professeur annonce que les élèves rédigeront en plusieurs temps : des descriptions dont il devront justifier la fonction puis un roman historique dans lequel ils inséreront ces descriptions ;

- en $\mathrm{B}$, la consigne est formulée en termes métalinguistiques et porte sur le nom et la phrase; en $\mathrm{H}$, le professeur choisit une métaphore, celle du puzzle, qui conduit à envisager le texte dans sa globalité et ses relations internes ;

- en $\mathrm{B}$, la référence à un texte lu est seulement thématique - il s'agit de transposer des personnages d'Harry Potter dans une scène de la vie quotidienne; en $\mathrm{H}$, le rapprochement avec le Chevalier an bouclier vert est à la fois thématique et fonctionnel, les élèves ayant lu le roman pour son apport documentaire et relevé les descriptions pour étudier leur fonction.

L'examen de ces différences ne conduit pas à proscrire toute consigne de réécriture formulée en termes métalinguistiques mais à cerner les conditions d'une utilisation possible : avec des élèves plus âgés, ayant acquis l'habitude de se décentrer par rapport à leur texte, dans une phase de relecture plutôt que de conception, une fois le projet d'écriture et les enjeux de la réécriture clarifiés. De telles consignes seraient utiles aux élèves de la séquence $H$, pour leur faire prendre conscience des ruptures syntaxiques produites par l'insertion de certaines descriptions.

Les données et les analyses ainsi présentées sont destinées aux enseignants en formation, afin qu'ils objectivent et confrontent des pratiques, mais elles s'adressent aussi aux chercheurs : en proposant un corpus non normé, où les textes convoquent sous des formes très diverses des représentations de l'oral et de l'écrit, textes pris dans la dynamique des médiations et des réécritures, il devient possible de cerner des processus qui interrogent à la fois la génétique textuelle, les travaux sur l'hétérogénéité discursive, ceux sur la spécificité de l'écrit et, sans l'y ramener, du littéraire. Pour revenir à la didactique en étudiant la façon dont des dispositifs d'écriture induisent chez les élèves des déplacements différenciés et inégalement investis subjectivement. 


\section{BIBLIOGRAPHIE}

BAUTIER E. \& BUCHETON D. (1995) «L'écriture : qu'est ce qui s'apprend? qu'est ce qui s'enseigne, qu'est-ce qui est déjà-là ? », Le Français Aujourd'bui n 111, pp. 26-35.

Benveniste, C.-B. (1997) Approches de la langue parlée en français, Paris, Ophrys.

Bonnet, C. (1994) Plume en main ou litinéraire de l'élève qui apprend à écrire, Centre vaudois de recherches pédagogiques.

Bonnet C., Corblin C. \& Elalouf M.-L. (1998) Les procédés d'écriture chez les élèves de 10 à 13 ans, un stade de développement, Lausanne, LEP, Loisirs et Pédagogie.

BONNET C. \& HuSER N. (1998) Une activité d'écriture en 8ème, correction, récriture et évaluation: les pratiques d'une enseignante, Lausanne, CVRP.

BORÉ, C. (2000) «Le brouillon, introuvable objet d'étude ? », Pratiques, 105-106, Metz, pp. 2349.

BORÉ, C. (2003) «Activités langagières en géométrie et en grammaire : de la définition à la notion ou l'inverse? ", Actes du Colloque de l'Université Victor-Segalen, 3-4-5 avril 2003 (cédérom édité par Martine Jaubert, Maryse Rebière et Jean-Paul Bernié).

BORÉ, C. (2003) «Reformulations orales et écrites : part du cognitif, part du linguistique », colloque INRP L'écriture et son apprentissage, questions pour la didactique, apports de la didactique, Paris 21-23 mars 2002, Repères 25-26, pp. 259-275.

J. Bouchard, J., De Gaulmyn, M.-M.\& Sadni-Jallab, R. (1998) «Impeccable! Le processus d'amélioration du texte en écriture coopérative» Cabier de linguistique française, 20, pp. 103-127.

BUCHETON D. (1997) Conduites d'écriture au collège et au lycée professionnel, CRDP de l'académie de Versailles.

David J., Plane, S. (1996) L'apprentissage de l'écriture. Paris, PUF.

Elalouf M-L, Keraven, J. (2002) «Une banque de données de textes d'élèves à l'épreuve », Pratiques, 115-116, pp. 107-124, Metz.

Elalouf, M-L, Keraven, J. (2003) «Ecrits exploratoires, écrits à contraintes. Quelle complémentarité en classe de $5^{e}$ ?", Littératures et langages, 1, Ecritures, pp. 149-156, CRDP de l'Académie de Versailles.

Elalouf, M-L, Keraven, J. (2004) «L'acquisition du lexique à l'épreuve d'un grand corpus d'élèves », Communication au colloque de mars 2003 Enseignement-apprentissage du lexique organisé par Lidilem (Grenoble 3), Ceditec (Paris 12) et Syled-Res (Paris 3) (à paraittre aux éditions de Boeck en 2004).

ElaLOUf M.-L., dir. (à paraître en 2004) Ecrire entre 10 et 14 ans, un corpus, des analyses, des repères pour la formation, CRDP de Versailles

FABRE-Cols, C., dir. (2000) Apprendre à lire des textes d'enfants, De Boeck-Duculot, Bruxelles.

GADET, F. (1989) Le français ordinaire, Paris, Armand Colin. 
Marie-Laure Elalouf

LAMOTHE-BOrÉ, C. (1998) Choix énonciatifs dans la mise en mots de la fiction, le cas des brouillons scolaires, Thèse de Doctorat de l'Université Stendhal-Grenoble 3, sous la direction de Claudine Fabre.

GRESILLON, A. (1994) Eléments de critique génétique. Lire les manuscrits modernes, Paris, PUF.

Masseron, C. (1989) «La correction de rédaction», in Pour une didactique de l'écriture, Metz, Pratiques, pp. 85-106,

Plane, S. (1998) «La production d'écrit s'enseigne-t-elle? Pratiques déclarées et représentations d'enseignants du second degré en France », Communication NTCE, Bordeaux.

Reuter, Y. (2000) La description. Des théories à l'enseignement-apprentissage. Paris, ESF. 